\title{
Transforaminal Endoscopic Discectomy: A Targeted Spinal Surgery
}

Ed Simor Khan Mor Japar Khan, Kow Ren Yi

Department of Orthopaedic Surgery, International Islamic University Malaysia.

Introduction: Open discectomy has been the gold standard of care for cases of Prolapsed intervertebral Disc. Tissue dissection is unavoidable in order to access the pathologic disc material. From skin incision, subcutaneous fat tissue, paraspinal muscles, ligamentum flavum, epidural fat, dura and nerve roots has to be encounter before reaching the disc material. With the in out transforaminal endoscopic technique only skin and subcutaneous tissue need to be bridge before reaching the disc material. This technique is also known as a targeted surgical technique. Minimal tissue injury is caused via this technique. Case report: We present 3 case series of prolapsed intervertebral disc which was treated with transforaminal endoscopic technique. Case 1: 49 year old presented with one year history of low back pain and left lower limb sciatica. Straight leg raising sign was positive. MRI showed 2 level disc bulge at L4L5, L5S1 with annular tear at L5S1. Case 2: 26 year old lady presented with 6 months history of low back pain and left lower limb sciatica. Straight leg raising sign was positive. MRI showed extruded disc at L4L5. Case 3: 28 year old lady presented with 8 months history of low back pain and left lower limb sciatica. Straight leg raising sign was positive. MRI showed extruded disc at L5S1 with annular tear. Results: All three patients underwent transformational discectomy. Case 1 and 2 were done under monitored anaesthesia control (MAC) and case 3 was done under epidural. All three patients had satisfactoryÂ pain relief with complete resolve of straight leg raising (SLR) test. The 1st patient had longer recovery period possibly because of its chronicity of symptoms. Conclusion: Early recovery is expected hence early return to work can be accomplish. In the future this might be the gold standard of treating prolapsed intervertebral disc. 